Few-Body Systems 4, 161-177 (1988)

\title{
Pairing Correlations: I. The Ground-State Coupled-Cluster Formalism as a Unifying Approach
}

\author{
R. F. Bishop, W. Piechocki*, and G. A. Stevens \\ Theoretical Physics Group, Department of Mathematics, University of Manchester Institute of Science \\ and Technology, P.O. Box 88, Manchester M60 1QD, England
}

\begin{abstract}
The general problem of pairing correlations within a many-fermion system of identical particles is discussed at some length. We show in this first work in a series how the ground-state version of the $[\exp (S)$ or $]$ coupled-cluster formalism (CCF) of quantum many-body theory may be rather generally applied to this problem, and how it thereby provides a very powerful and unifying approach to it. At the so-called SUB2 level of truncation, which is the lowest natural level of approximation for homogeneous systems, the CCF may be cast as a nonlinear integral equation for a four-point correlation function, $S_{2}$, which provides a measure of the two-particle/two-hole component in the true "ground-state" wavefunction. This approximation couples the particle-particle, hole-hole and particle-hole correlations simultaneously, and treats them all on an equal footing. In the present work we concentrate particular attention on particle-particle and hole-hole correlations by focussing on the formulation of generalised ladder approximations within the CCF. These are therefore likely to be physically applicable in the low-density regime. In particular, we show that the well-known Galitskii approximation may be formulated within the CCF as that drastic sub-approximation to the full SUB2 equation which keeps only the so-called complete ladder (CLAD) terms, which we describe. A second paper applies the CCF to the particular case of a general (non-local) separable potential, and obtains exact analytic solutions within the CLAD approximation for the corresponding wavefunctions of the simultaneous particle-particle and hole-hole substructures within the many-fermion system.
\end{abstract}

\section{Introduction}

Many-body calculations on the scattering and binding properties of constituent pairs of fermions imbedded in a large, typically infinite, background of identical particles, have historically played a crucial role both in understanding the observed properties of many condensed matter systems [1], and also for developing and

\footnotetext{
* On leave of absence from: Nuclear Theory Department, Institute for Nuclear Studies, Hoża 69, PL-00-681 Warsaw, Poland
} 
understanding the various formulations of many-body theory itself. Furthermore, bound pairs within the many-body medium serve as the simplest example of the more general problem of the existence and nature of bound clusters within manybody systems, and the resultant effects of such clustering on the macroscopic properties of these systems.

Typically, for the case of pairing, an effective two-body scattering amplitude ( $G$-matrix, $T$-matrix, etc.) is first defined as the key element of one type of microscopic theory. Although not amenable to direct physical observation, such a pair amplitude is often then used in turn either in phenomenological descriptions as an effective stepping-stone in relating thermodynamic and average single-particle behaviour [2]; or in microscopic perturbation theories as a lowest-order buildingblock in a useful, if not even vital, rearrangement of the perturbation series [3]. Perhaps the most well-known and one of the most successful applications of such concepts was to the phenomenon of superconductivity, where an analysis of the scattering of electron pairs in a degenerate electron gas (the Cooper problem) [4], showed how the phenomenon could be explained via the formation of bound electron pairs within the many-electron system.

Pairing correlations within a many-body system have most frequently been discussed in the past within the framework of many-body perturbation theory for the ground-state (g.s.) energy. On the one hand there have been the calculations within the time-independent perturbation formalism which can be conveniently represented via the linked-cluster perturbation series of Goldstone [3] in the diagrammatic language of (time-ordered) Goldstone diagrams. On the other hand similar calculations have been performed within time-dependent perturbation theory, for which the usual starting-point is the Green's function formalism of Galitskii and Migdal [5], which in turn grew out of the analogous techniques developed by Feynman for relativistic quantum field theory, where the associated non-time-ordered diagrams are generally known as Feynman diagrams. Both the time-independent and time-dependent formulations of perturbation theory provide equally valid calculational frameworks, which would give identical (exact) results if carried out to infinite order. In practice of course both must be truncated, at their respective pair correlation levels for example, and in this case they are not identical. At the level of pair correlations, equivalent to their (different) respective ladder approximations, discussed in more detail below, the connections between and the relative merits of the two approaches have been discussed by one of the present authors [6] for the case of calculations of the g.s. energy of (dilute) many-fermion systems.

For most realistic many-body systems of interest, it is also the case that the interparticle interactions are either too singular or too strong to permit a straightforward perturbation expansion in powers of the interaction potential. In typical cases of physical interest the pair potential is likely to become strongly repulsive at small interparticle separations, thereby ensuring that for any physical quantity (such as the g.s. energy), an expansion based on counting the number of pairwise interactions is likely to converge only very slowly at best. In such cases, Brueckner $[1,7]$ first realised within the many-body context that the correct mathematical treatment of this problem was to sum into a single term (namely the so-called two-body $T$-matrix or $G$-matrix or one of their counterparts) each of the infinite number of distinct terms in the original series (defined in terms of powers of 
the two-body potential) that involves only successive interactions between a given pair of particles, with the remaining particles acting only as spectators. In diagrammatic language, the terms thus defined are the well-known two-body ladder diagrams. The original perturbation series in terms of the two-body interaction potential, $V$, may finally (at least in principle) be exactly rearranged to eliminate $V$ in favour of the exact two-body scattering amplitude (or $T$-matrix, etc.) previously defined. The hope then is that at least under suitably well-defined physical conditions, the resultant series may exhibit much better convergence properties than the original series.

The ladder diagrams that describe the repeated interactions (in a perturbationtheoretic sense) between the same pair of bodies within the many-body medium can be isolated and defined within both the time-independent and time-dependent formulations of many-body perturbation theory. In the time-independent formalism this ladder summation describes the scattering between two fermions outside the Fermi sea, and by a well-known procedure the corresponding $T$-matrix can be written as the solution to the Bethe-Goldstone (BG) equation [8]. In the timedependent formalism, the particles (outside the Fermi sea) and holes (inside the Fermi sea) are treated completely symmetrically, and the analogous equation for the corresponding $T$-matrix [which we shall henceforth refer to as the GalitskiiFeynman (GF) $T$-matrix] is now the (ladder approximation to the) Bethe-Salpeter equation [9]. The GF T-matrix thus sums as Feynman diagrams, the same two-body diagrams which the $\mathrm{BG}$ method treats as Goldstone diagrams. In other words, considered wholly in terms of time-ordered Goldstone diagrams, the GF T-matrix incorporates not only all the BG particle-particle ladders, but also all the hole-hole and mixed particle-particle and hole-hole ladders generated from them only by changing the time-orderings of the various interactions through the two-body potential $V$. The intermediate scattering states in this case thus comprise (arbitrary numbers of) both two-particle and two-hole pairs.

Comparisons between the various generalised ladder approximations have a quite long history [10-12], and much attention has been focussed in particular on the existence and energies of the various bound pair states possible in each. The difference between the BG and GF approaches, which is accounted for by the latter treating particles and holes symmetrically and thus including hole-hole scattering and mixed particle-particle/hole-hole scattering as well as particle-particle scattering, was perhaps first demonstrated to be important in the case of realistic potentials for both nuclear matter and liquid ${ }^{3} \mathrm{He}$ by one of the present authors and his coworkers $[13,14]$. For both systems they showed that the GF $T$-matrix has singularities in the form of first-order poles in the (off-shell) energy variable in the centre-of-mass frame, which are absent in the corresponding BG $T$-matrix. The existence of these poles furthermore corresponds to the formation of bound-state pairs, as expected by analogy with the two-body $t$-matrix solution to the fully offshell Lippmann-Schwinger equation [15] which describes two-body scattering in vacuo, and to which the BG and GF $T$-matrices must tend as the density of the many-body system tends to zero. Numerical calculations on these singularities were also performed $[13,14,16]$, specifically for the cases of liquid ${ }^{3} \mathrm{He}$ and nuclear matter using typical phenomenological He-He and nucleon-nucleon interaction potentials respectively, and more general results were also given later for the structure of composite pairs and effective two-body scattering within a many-body 
medium, using the GF formalism [17]. Exact analytic results have also been given for both the BG [18] and GF [19] $T$-matrices for the soluble model interactions of the pure hard-sphere (or hard-core) interaction, and its generalisation to the boundary-condition model $[20,21]$. The role of the hole states in binding quasiparticle pairs within the many-body medium was particularly demonstrated in ref. [19], where it was shown that such bound pairs can exist even when the bare potential supports no free (i.e., in vacuo) two-body bound states or also when there is no possibility of bound BG pairs. A particularly vivid illustration was given of how the inclusion of hole-hole scattering can in principle dramatically alter the properties of a many-body system even at arbitrarily low densities, in contradiction to quite widely held views to the contrary.

From the physical viewpoint the existence of bound composite clusters of fermions within a many-fermion system is of considerable importance, since under suitable conditions any such even complexes or composite bosons may condense, leading to a phase transition in the system. In this respect it is interesting to note that Kohn and Sherrington [22] have classified composite bosons into two types, namely Type I which are comprised of complexes of an even number of real fermions (particles) and/or an even number of their corresponding holes; and Type II which are bound complexes of particle-hole pairs, more generally known as "collective excitations". Examples of Type I bosons are ${ }^{4} \mathrm{He}$ atoms and Cooper pairs, while examples of Type II bosons are phonons, plasmons, excitons, and the giant resonances in atomic nuclei. The important point made by Kohn and Sherrington is that when a system of Type I bosons condenses there results a state which is ordered in momentum space in the terminology of London, or equivalently in the more modern terminology of Yang [23], exhibits off-diagonal long-range order (ODLRO). Such ODLRO then leads to a superfluid-like phase. Conversely, when a system of Type II bosons condenses, the resultant state does not exhibit ODLRO although there is a change of long-range order in real (coordinate) space, i.e. there develops a diagonal long-range order (DLRO). Typical examples are the condensation of the Type I Cooper pairs which lead to the superconducting phase; and the condensation of the Type II excitons in the so-called excitonic phase of a photoexcited semiconductor wherein the excited electron-hole droplets become unstable against the formation of a coherent condensate of bound electron-hole pairs (or excitons). Another perhaps less familiar example of a Type II condensation is when a macroscopic number of phonons in a crystal Bose-condense into a single mode, which leads to a distortive phase transition of the crystal with the appearance of a superlattice.

Clearly, in our previous introductory discussion on pairing via the BG or GF formalisms (or any of the generalised particle-particle and hole-hole ladder schemes) we have restricted our analysis solely to Type I bosons of the composite fermion-pair variety. One of the most interesting general problems in modern quantum many-body theory is the interplay between and the mutual effects on each other of the many-body collective excitations and other collective aspects of the system's behaviour on the one hand, with the low-lying single-particle, two-particle and few-particle excitations and other few-particle aspects on the other hand. In the above language we can now exemplify one aspect of this problem as the interplay between the two types of composite bosons formed from the individual fermions and their holes. Since it is also generally true that long-range interactions tend to 
accentuate the collective excitations whereas short-range interactions tend to accentuate the few-body excitations, the general problem can also be broadly interpreted in terms of the interplay between the long and short-range interactions and correlations, both of which seem to be present to a comparable degree for such realistic systems of interest as liquid ${ }^{3} \mathrm{He}$ and nuclear matter. In both cases the interparticle potential is characterised by strong short-range repulsion followed by a weaker but long-ranged attractive tail.

One of the main aims of the present work is to imbed the above problem in a many-body formalism which is both rich enough and powerful enough to encompass all aspects of it, and which we hope to demonstrate is uniquely equipped to provide practical solutions. The formalism that we propose to use is the $\exp (S)$ method as it was originally called or, to give it its more modern name, the coupled-cluster formalism (CCF) of Coester and Kümmel [24-28]. In the present work, which is intended to be the first in a new series, we intend to focus initial attention on Type I pairing by formulating the generalised particle-particle/holehole "ladders" within the CCF, firstly in the ground-state (g.s.) scheme. A second companion paper [29] applies the formalism to a model potential of the separable kind. A later third paper [30] will extend the discussion to the excited-state (e.s.) scheme, and in subsequent work we intend to incorporate also all aspects of particlehole pairing (both through the particle-hole "bubbles" and the particle-hole ladders), self-consistent particle- and hole-potential (or self-energy) effects, exchange (antisymmetry), and other effects elucidated more clearly below.

In this spirit the present series of works can also be viewed as forming a complementary series to that already given by one of the present authors and his coauthor [31-35], which addressed essentially the same problems but by focussing initial attention in the first paper [31] (hereafter referred to as I) on Type II pairing by first formulating within the CCF the generalised particle-hole "bubble" terms. These terms are of particular importance for example to the electron correlations in the one-component Coulomb plasma (alias the "electron gas" or "jellium"), where they provide the asymptotically leading contribution to the g.s. energy as the density tends to infinity. In particular, exact analytic results were given in I for the case of a local two-body potential for a hierarchy of three bubble approximations which occur naturally within the CCF, namely the so-called Macke approximation (MA) [36], the Tamm-Dancoff approximation (TDA) [37], and the random-phase approximation (RPA) [38, 39]. In the second paper of that series [32] (hereafter referred to as II), the full power of the CCF was brought to bear on the g.s. correlations in the one-component Coulomb plasma at low and metallic densities with remarkable success.

The present paper and its sequel [29] should be viewed as the analog for particleparticle and hole-hole ladder terms of what I was for the particle-hole bubble terms. Our main aim in these first two papers is again to obtain exact analytic results both because such results are rare (and hence valuable) in many-body theory and also because they provide a yardstick against which future approximations can be measured, just as the results of I were invaluable for later use in II. For this reason alone we consider applications in the succeeding paper [29] to (non-local) separable two-body interactions, just as I considered only local interactions. It is perhaps worth noting at this point that although we restrict ourselves for ease of discussion to one-term $S$-wave interactions, the extension to both multi-term separable inter- 
actions and to arbitrary higher partial waves is straightforward. Hence what may appear at first sight to be a rather artificial or non-physical interaction, can in practice rather easily be extended to give a very good approximation to an arbitrary, even local, potential by choosing a sufficient number of separable terms in each partial wave. We shall also see that there is a quite remarkable similarity between the results for local interactions of the triad of bubble approximations already mentioned, with those for separable interactions for another hierarchy of three ladder approximations that also arises naturally within the CCF. These are analogous respectively to (i) the BG approximation with its associated particleparticle ladders (PPLAD); (ii) an intermediate approximation which adds to PPLAD the hole-hole ladders (HHLAD), and which seems first to have been investigated by Chisholm and Squires [10] and Mehta [11] who solved for the respective $T$-matrix in the case of separable interactions; and finally (iii) the addition to PPLAD and HHLAD of the mixed particle-particle/hole-hole ladders (MLAD) to give the so-called complete ladder (CLAD) sum of the GF approach. This latter CLAD approximation has also been investigated for separable interactions by Mehta [12] who gave results for the pairing energies but did not solve for the respective $T$-matrix or any analogous four-point function in this case.

Since the CCF has been discussed for present purposes in some detail in I to which the reader is directed both for a self-contained formulation sufficient for this work, and for references to other applications of the formalism), we restrict ourselves here to a brief introductory review of the physical content of the g.s. formalism. The linked cluster theorem for the exact many-body ground-state wavefunction [i.e., the usual $\exp (S)$ ansatz] is first used to decompose the $N$-body system formally into a set of mutually interacting $n$-body subsystems $(n \leqslant N)$. Each subsystem is thereby fully described by its own so-called correlation operator $S_{n}$, which has non-vanishing matrix elements only between states comprising $n$ particles on the one hand and $n$ holes on the other. Formally the theory is "linked" in the sense that "disconnected" terms are eliminated (for the g.s. energy, for example), precisely because the $S_{n}$ operators have vanishing matrix elements between any two $n$-body states that both contain any particle label or both contain any hole label. The (only non-zero) matrix elements of the correlation operators thus describe the excitation of $n$ particle-hole pairs from some suitably defined, but otherwise quite arbitrary, $N$-body reference state or model state (often chosen to be, or referred to as, the noninteracting state). The exact g.s. $N$-body Schrödinger equation is thus easily decomposed into a coupled set of $N$ (nonlinear) microscopic equations for the correlation amplitudes (i.e., the matrix elements of the operators $S_{n}$ ). The elimination of the unlinked terms, formally achieved by the $\exp (S)$ ansatz, then guarantees that each of the microscopic equations contains no macroscopic term (i.e., any term proportional to $N$ ).

The general structure of this set of equations is that the $n$-th equation in the hierarchy for $S_{n}$ is coupled, in the general case where the many-body Hamiltonian contains up to $j$-body interaction terms, to the other amplitudes $S_{i}$ with $1 \leqslant i \leqslant(n+j)$. For our purposes we shall be interested only in two-body interparticle forces, so that $j=2$. In order for this exact decomposition to be of use, this hierarchy of equations must be truncated in a physically meaningful way, and here it is clearly necessary, although not sufficient, that any macroscopic terms must already have been eliminated. The truncation scheme employed in I, and largely also 
in II, and with which we shall be wholly concerned also here, is the so-called "natural" scheme or $\mathrm{SUB} n$ scheme in which all operators $S_{i}$ for $i>n$ are set to zero, and the remaining $n$ coupled nonlinear equations are solved for the matrix elements of the operators $S_{m}$ with $1 \leqslant m \leqslant n$. It is worth pointing out at this point that the proposed truncation scheme leads to a hierarchy of approximations which asymptotically become exact in a monotonic fashion [i.e., the SUB $n$ approximation is guaranteed to be better than the $\operatorname{SUB}(n-1)$ approximation]. Furthermore, and importantly for the present work where attention is focussed on generalised pairing, at each level of approximation the formalism treats the particle and hole states in a completely symmetric fashion.

The SUB1 approximation is fully equivalent to the well-known Hartree-Fock approximation. In the case of infinite homogeneous and isotropic systems, with which we shall largely be concerned, this approximation becomes trivial when the reference state is chosen to be the noninteracting state (i.e., the filled Fermi sea or Slater determinant of plane-wave states), since then $S_{1} \equiv 0$ by momentum conservation. The approximation to which we therefore direct our attention in the present series of works is the SUB2 approximation, which was discussed in detail in I and which is summarised in the next section. For the purposes of a preliminary orientation we point out firstly that the fermion SUB2 approximation contains as drastic sub-approximations to itself, such other approximations as we have already discussed, namely (i) the RPA, and indeed the other particle-hole bubble approximations already mentioned, namely the MA and TDA, as further truncations of the terms which generate RPA; (ii) the GF approximation which sums the complete ladder (CLAD) terms describing particle-particle/hole-hole scattering, and indeed also the other ladder approximations already mentioned, including the BG approximation (PPLAD terms only) and the intermediate approximation which treats the PPLAD and HHLAD terms simultaneously but without the mixed ladder (MLAD) terms between them. In fact, the SUB2 approximation goes beyond even the self-consistent union of the above approximations by also including (again in a completely integrated and fully self-consistent fashion) the terms which by themselves generate (iii) the additional RPAEX exchange terms necessary to antisymmetrise the RPA; the self-energy correction terms which yield both (iv) a self-consistent particle potential and, more importantly, (v) a self-consistent hole potential; (vi) repeated interactions between particle-hole pairs in the form of particle-hole ladders; and (vii) classes of extra higher exchange terms to preserve overall antisymmetry. We note in passing that the whole of the usual BruecknerBethe-Goldstone theory applied so successfully to nuclear matter, finite nuclei, and other Fermi systems is also included as a sub-approximation to SUB2 by keeping only the PPLAD terms and the hole potential terms (and where the "standard choice" of setting the particle potential to zero is usually made, after Brandow [40]).

We stress again that the basic SUB2 approximation is exact apart from ignoring the interactions with higher subsystems. In particular it includes all two-body effects (including both Type I and Type II pairing simultaneously and self-consistently, although of course it does not contain such higher order clustering as, for example, the Type I $\alpha$-particle clustering in nuclear matter, which would only be properly treated at the SUB4 level of approximation). It is not however surprising therefore that the SUB2 equations are complex, but we have already shown in both I and II that they are both physically transparent and mathematically tractable. Our 
emphasis in I was on systems with long-range interactions and Type II pairing, where the RPA terms dominate at high density, and we explored in II the effects on these of the short-range interactions and correlations through a systematic inclusion of the other terms in the full SUB2 approximation (and indeed beyond) which become of increasing importance as the density is lowered. We now, correspondingly, wish to focus attention more on systems with strong short-range interactions and Type I pairing, where we expect the various particle-particle and hole-hole ladder approximations to dominate at least at low density. Armed with the exact results from this work and its sequel [29], we then aim in a future work to investigate the effects of the other SUB2 terms (and beyond), on the pairing in particular, as the density is increased.

As an indication of the power and accuracy of the SUB2 approximation we mention only that for the one-component Coulomb plasma of fermions, the results in I and II for the g.s. energy were seen (i) to be exact in the high-density (weakcoupling) limit; (ii) to be accurate to about the $1 \%$ level in the difficult (intermediatecoupling) regime of metallic densities, by comparison with some recent essentially exact (Green's function Monte Carlo) stochastic simulations of the many-body Schrödinger equation; and (iii) to provide even in the (strong-coupling) low-density limit, a good "translationally-invariant solid" (i.e. amorphous or glassy solid) description in this exact Wigner crystal limit. Furthermore, all of these results were obtained in a single calculation which included even the indication of the phase transition (although of course the symmetry-breaking at the transition from a plasma to a crystal could not be observed since the wavefunction was constrained from the outset to be translationally-invariant). There is no doubt that the SUB2 results for this model system, which is itself probably the most well-studied of all quantum many-body systems, provide the currently best available microscopic description of at least its g.s. energy; and it is this success that provides strong motivation for the present study.

In Sect. 2 we give a brief formulation of the CCF, mainly to establish notation and terminology, and otherwise referring to I for details. We also focus in particular on the formulation within the CCF of the various generalised ladder approximations to which we have already alluded. In the succeeding paper [29] we then solve for the PPLAD (corresponding to BG approximation) and CLAD (corresponding to Galitskii approximation) cases in particular, obtaining exact results for the four-point function $S_{2}$ in both cases. The results obtained are discussed in ref. [29], where a particular comparison is made of the results for the (Type I) composite-pair states that emerge from the calculations. A brief further discussion of the CCF is given in Sect. 3, which concludes the present paper.

\section{Coupled Cluster Formalism and Ladder Approximations}

We consider a system of $N$ identical fermions that interact via the nonrelativistic Schrödinger equation with a Hamiltonian consisting of kinetic energies and twobody potentials $V$,

$$
\begin{gathered}
H=H_{0}+V, \\
H_{0}=\sum_{\alpha} \varepsilon_{\alpha} a_{\alpha}^{\dagger} a_{\alpha},
\end{gathered}
$$




$$
V=\frac{1}{(2 !)^{2}} \sum_{\alpha \beta \gamma \delta}\langle\alpha \beta|V| \gamma \delta\rangle_{A} a_{\alpha}^{\dagger} a_{\beta}^{\dagger} a_{\delta} a_{\gamma}
$$

where the operators $a_{\alpha}^{\dagger}$ are a set of fermion creation operators for the complete and orthonormalised single-particle (s.p.) basis states $|\alpha\rangle=a_{\alpha}^{\dagger}|0\rangle$, with $|0\rangle$ the vacuum state. In Eq. (2.1) the quantities $\varepsilon_{\alpha}$ represent the s.p. kinetic energies, and we have introduced our notation that $\left|\alpha_{1} \ldots \alpha_{n}\right\rangle$ represents a direct product state,

$$
\left|\alpha_{1} \ldots \alpha_{n}\right\rangle=\left|\alpha_{1}\right\rangle \ldots\left|\alpha_{n}\right\rangle
$$

and the subscript $A$ on the ket states denotes explicit antisymmetrisation (without normalisation) of that state, i.e.

$$
\begin{aligned}
\left|\alpha_{1} \ldots \alpha_{n}\right\rangle_{A} & =\sum_{P\{\alpha\}}(-1)^{P_{\alpha}}\left|\alpha_{1} \ldots \alpha_{n}\right\rangle \\
& =a_{\alpha_{1}}^{\dagger} \ldots a_{\alpha_{n}}^{\dagger}|0\rangle
\end{aligned}
$$

where the sum extends over all ( $n !)$ permutations of the indices $\alpha_{1} \ldots \alpha_{n}$, and $P_{\alpha}$ is the signature of the permutation.

The coupled cluster formalism (CCF) of many-body theory has already been discussed in some detail in I. In terms of a suitable model, or uncorrelated, $N$-fermion wavefunction $|\Phi\rangle$, the (usual linked cluster) ansatz for the exact, correlated $N$-fermion ground-state (g.s.) wavefunction $|\Psi\rangle$ is made,

$$
|\Psi\rangle=e^{S}|\Phi\rangle
$$

and we consider $|\Phi\rangle$ normalised to $|\Psi\rangle$ by $\langle\Phi \mid \Psi\rangle=1$. For present purposes we deal only with model Fermi states of single Slater determinant form,

$$
|\Phi\rangle=a_{v_{1}}^{\dagger} \ldots a_{v_{N}}^{\dagger}|0\rangle \text {. }
$$

Although it is obviously possible to consider more general s.p. basis sets, it is important for later discussions to realise that for all the results reported here, we deal exclusively with plane-wave s.p. states, appropriate to homogeneous and isotropic, infinite systems. Considering a system of identical spin- $\frac{1}{2}$ fermions (- the extension to include isospin, for example, is trivial), we then have in coordinate space representation,

$$
\langle\vec{r} \mid \alpha\rangle=\Omega^{-1 / 2} \exp \left(i \vec{k}_{\alpha} \cdot \vec{r}\right) \eta\left(s_{\alpha}\right),
$$

with $\eta\left(s_{\alpha}\right)$ the two spin-functions for spin-up and spin-down along a chosen projection axis, and $\Omega$ is the volume of the periodicity box. The s.p. kinetic energies are

$$
\varepsilon_{\alpha}=\hbar^{2} k_{\alpha}^{2} / 2 m
$$

where $m$ denotes the particle mass. The non-interacting g.s. $|\Phi\rangle$ is now the usual filled Fermi sea in which all s.p. states with momenta smaller than the Fermi momentum $\hbar k_{F}$, are occupied. Henceforth we use the standard notation that s.p. labels $v_{i}$ indicate states "normally occupied" in $|\Phi\rangle\left(\right.$ i.e., with $k_{v_{i}}<k_{F}$ ) and which hence later refer to "hole states", s.p. labels $\rho_{i}$ indicate states outside the Fermi sea (i.e., "normally unoccupied" or "particle" states), and s.p. labels $\alpha_{i}$ (and $\beta, \gamma, \delta$ ) run over all states. 
The correlation operator $S$ of Eq. (2.2) is decomposed into $n$-body $(n \leqslant N)$ components,

$$
\begin{gathered}
S=\sum_{n=2}^{N} S_{n}, \\
S_{n}=(n !)^{-2} \sum_{\substack{\rho_{1} \ldots \rho_{n} \\
v_{1} \ldots v_{n}}} a_{\rho_{1}}^{\dagger} \ldots a_{\rho_{n}}^{\dagger}\left\langle\rho_{1} \ldots \rho_{n}\left|S_{n}\right| v_{1} \ldots v_{n}\right\rangle_{A} a_{v_{n}} \ldots a_{v_{1}},
\end{gathered}
$$

and the usual linked-cluster aspect is expressed by Eqs. (2.2) and (2.6) in which the only matrix elements of the correlation operators $S_{n}$ that enter are the real $n$-particle $/ n$-hole matrix elements. It is also important to realise that the sum in Eq. (2.5) omits the term $n=1$ by momentum conservation, but only as a consequence of our implied assumption that the exact g.s. wavefunction $|\Psi\rangle$ is also an eigenstate of total momentum (with eigenvalue zero). It is further explained in some detail in I how the matrix elements of the operators $S_{n}$ represent the true $n$-body correlations after all the factorisable (or unlinked) correlations have been removed from the complete $n$-body subsystem amplitudes,

$$
\left\langle\alpha_{1} \ldots \alpha_{n}\left|\Psi_{n}\right| v_{1} \ldots v_{n}\right\rangle_{A} \equiv\left\langle\Phi\left|a_{v_{1}}^{\dagger} \ldots a_{v_{n}}^{\dagger} a_{\alpha_{n}} \ldots a_{\alpha_{1}}\right| \Psi\right\rangle .
$$

Formally, the g.s. CCF now proceeds by decomposing the $N$-body Schrödinger equation,

$$
H|\Psi\rangle=E|\Psi\rangle
$$

into a coupled set of equations for the matrix elements of the correlation operators $S_{n}$. Formally this may be achieved by combining Eqs. (2.8) and (2.2) into the form

$$
e^{-S} H e^{S}|\Phi\rangle=E|\Phi\rangle
$$

and then taking the overlap of Eq. (2.9) with the state $\langle\Phi|$ or with the $n$-particle/ $n$-hole states $\langle\Phi| a_{v_{1}}^{\dagger} \ldots a_{v_{n}}^{\dagger} a_{\rho_{n}} \ldots a_{\rho_{1}}$ built on it with $n \leqslant N$. This procedure has been discussed in some detail in I, where the resulting equations for $n=(1$ and $) 2$ have been derived. An alternative but completely equivalent approach has also recently been given by Dabrowski [41], which is based on an analysis of the structure of the equivalent Goldstone diagrams for the wavefunction of the system.

The above procedure leads to a coupled set of equations for the matrix elements of $S_{n}$ in which the $i$-th equation (for $S_{i}$ ) is coupled both to $S_{i+1}$ and $S_{i+2}$ (as well as to all $S_{j}$ with $j<i$ ) for a Hamiltonian like Eq. (2.1) that involves up to two-body potentials only. Clearly, in order to be useful this exact coupled hierarchy has to be truncated, and one such approximation scheme has been discussed in I, namely the so-called SUB $n$ approximation scheme in which one sets $S_{i}=0$ for all $i>n$, and the remaining equations (for given $n$ ) are treated as accurately as possible. We have already alluded in Sect. 1 to the terms contained in the SUB2 approximation, with which we shall be concerned here, and we now refer both to Eqs. (2.12)-(2.27) of I for the complete analytic form of the SUB2 equation in this case, and also to Fig. 1 of I for its diagrammatic representation.

We note that although it can formally be shown [42] how to obtain quantities equivalent to those expressible by the time-ordered Goldstone diagrams of timeindependent perturbation theory, this equivalence is now intuitively almost obvious. Thus, when one realises that a Goldstone diagram for the wavefunction, for 
example, is always referred to the state $|\Phi\rangle$, and it is always implied that all of the non-participating particles, corresponding to the "missing" lines in the diagram, are in their "normally occupied" s.p. states, the analogy with the matrix elements $\left\langle\left|\Psi_{n}\right|\right\rangle$ of Eq. (2.7), and hence via Eq. (2.2) with the matrix elements $\left\langle\left|S_{n}\right|\right\rangle$, is then transparent. Conversely, we also note that the equivalence of the present CCF with the Green's function approach based on the Feynman-Dyson time-dependent perturbation theory, is more complicated. This latter formalism can also be expressed diagrammatically in terms of non-time-ordered or Feynman diagrams, but these have no direct counterpart in our approach.

Starting from the SUB2 approximation for $S_{2}$ we now wish, as already discussed in Sect. 1, to retain only those terms responsible for generating the complete sum of ladder diagrams for two-particle/two-hole scattering in the many-body medium, i.e. the particle-particle, hole-hole, and mixed particle-particle and hole-hole ladders. This leads us to what we shall call the complete ladder (CLAD) equation. It should be clear from our previous discussion that this CLAD equation is completely equivalent to the GF $T$-matrix approach. The CLAD approximation may be formally arrived at by keeping only the first two terms on the right-hand side of the otherwise exact Eq. (2.10) of I for $S_{2}$, and further by keeping only (bare) kinetic energies for the hole energy terms given by Eq. (2.11) of I in the left-hand side of Eq. (2.10) of I. In our compact notation, the basic CLAD equation may then be written as

$$
\begin{aligned}
\left(\varepsilon_{\rho_{1}}\right. & \left.+\varepsilon_{\rho_{2}}-\varepsilon_{v_{1}}-\varepsilon_{v_{2}}\right)\left\langle\rho_{1} \rho_{2}\left|S_{2}\right| v_{1} v_{2}\right\rangle_{A} \\
& =-\left\langle\rho_{1} \rho_{2}\left|V \Psi_{2}\right| v_{1} v_{2}\right\rangle_{A}-\frac{1}{2} \sum_{v v^{\prime}}\left\langle\rho_{1} \rho_{2}\left|S_{2}\right| v v^{\prime}\right\rangle_{A}\left\langle v v^{\prime}\left|V \Psi_{2}\right| v_{1} v_{2}\right\rangle_{A} .
\end{aligned}
$$

As in I, this CLAD equation (2.10) may now be considered more fully in our planewave basis (2.4). Restricting ourselves henceforth to a spin-independent pairwise potential, and making explicit use of both conservation of total momentum and of projected component of total spin, Eq. (2.10) becomes

$$
\begin{aligned}
\frac{\hbar^{2}}{2 m}\left(\mid \vec{k}_{1}\right. & \left.+\left.\vec{q}\right|^{2}+\left|\vec{k}_{2}-\vec{q}\right|^{2}-k_{1}^{2}-k_{2}^{2}\right)\left\langle\vec{k}_{1}+\vec{q}, s_{1} ; \vec{k}_{2}-\vec{q}, s_{2}\left|S_{2}\right| \vec{k}_{1} s_{1} ; \vec{k}_{2} s_{2}\right\rangle_{A} \\
= & -\left\langle\vec{k}_{1}+\vec{q} ; \vec{k}_{2}-\vec{q}|V| \vec{k}_{1} \vec{k}_{2}\right\rangle+\left\langle\vec{k}_{1}+\vec{q} ; \vec{k}_{2}-\vec{q}|V| \vec{k}_{2} \vec{k}_{1}\right\rangle \delta_{s_{1} s_{2}} \\
& -\sum_{\vec{q}^{\prime}}\left\langle\vec{k}_{1}+\vec{q} ; \vec{k}_{2}-\vec{q}|V| \vec{k}_{1}+\vec{q}^{\prime} ; \vec{k}_{2}-\vec{q}^{\prime}\right\rangle \\
& \times\left\langle\vec{k}_{1}+\vec{q}^{\prime}, s_{1} ; \vec{k}_{2}-\vec{q}^{\prime}, s_{2}\left|S_{2}\right| \vec{k}_{1} s_{1} ; \vec{k}_{2} s_{2}\right\rangle_{A} \bar{n}\left(\vec{k}_{1}+\vec{q}^{\prime}\right) \bar{n}\left(\vec{k}_{2}-\vec{q}^{\prime}\right) \\
& -\sum_{\vec{k}}\left\langle\vec{k}_{1}+\vec{q}, s_{1} ; \vec{k}_{2}-\vec{q}, s_{2}\left|S_{2}\right| \vec{k}_{1}+\vec{k}, s_{1} ; \vec{k}_{2}-\vec{k}, s_{2}\right\rangle_{A} \\
& \times\left\langle\vec{k}_{1}+\vec{k} ; \vec{k}_{2}-\vec{k}|V| \vec{k}_{1} \vec{k}_{2}\right\rangle n\left(\vec{k}_{1}+\vec{k}\right) n\left(\vec{k}_{2}-\vec{k}\right) \\
& -\left(1-\frac{1}{2} \delta_{s_{1} s_{2}}\right) \sum_{\vec{k} \vec{q}^{\prime}}\left\langle\vec{k} \vec{k}_{1}+\vec{q}_{1}, s_{1} ; \vec{k}_{2}-\vec{q}, s_{2}\left|S_{2}\right| \vec{k}_{1}+\vec{k}, s_{1} ; \vec{k}_{2}-\vec{k}, s_{2}\right\rangle_{A} \\
& \times\left\langle\vec{k}_{1}+\vec{k} ; \vec{k}_{2}-\vec{k}|V| \vec{k}_{1}+\vec{q}^{\prime} ; \vec{k}_{2}-\vec{q}^{\prime}\right\rangle \\
& \times\left\langle\vec{k}_{1}+\vec{q}^{\prime}, s_{1} ; \vec{k}_{2}-\vec{q}^{\prime}, s_{2}\left|S_{2}\right| \vec{k}_{1} s_{1} ; \vec{k}_{2} s_{2}\right\rangle_{A} \\
& \times n\left(\vec{k}_{1}+\vec{k}\right) n\left(\vec{k}_{2}-\vec{k}\right) \bar{n}\left(\vec{k}_{1}+\vec{q}^{\prime}\right) \bar{n}\left(\vec{k}_{2}-\vec{q}^{\prime}\right)
\end{aligned}
$$




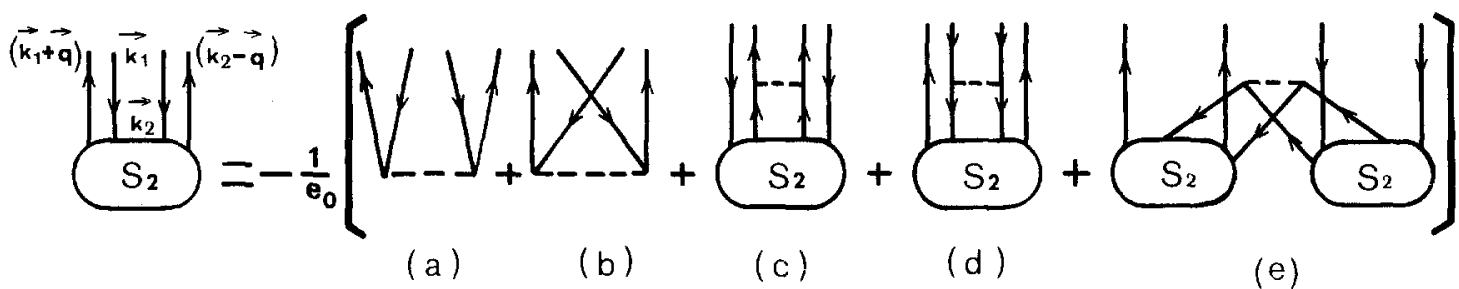

Fig. 1. Diagrammatic representation of the CLAD equation for $S_{2}$. The dashed line represents the bare potential $V$, and $e_{0}$ is given by Eq. $(2.13)$

where the functions $n(k)$ and $\vec{n}(k)$ are respectively the unperturbed hole and particle occupation probabilities, given in terms of the usual unit-step function $\theta(x)$ defined to be one (zero) for $x$ greater (less) than zero, by

$$
n(k) \equiv \theta\left(k_{F}-k\right), \quad \bar{n}(k) \equiv \theta\left(k-k_{F}\right) .
$$

Equation (2.11) thus corresponds to choosing out of the full SUB2 equation (2.15) of I only the term $T_{\text {CLAD }}$ (given by Eq. (2.23) of I) apart from the kinetic energy terms and the inhomogeneous, driving (bare direct potential and its exchange) terms previously lumped into the $T_{\mathrm{RPA}}$ and $T_{\mathrm{RPAEX}}$ terms in I. By dividing throughout by the kinetic energy term,

$$
\frac{\hbar^{2}}{2 m}\left(\left|\vec{k}_{1}+\vec{q}\right|^{2}+\left|\vec{k}_{2}-\vec{q}\right|^{2}-k_{1}^{2}-k_{2}^{2}\right) \equiv e_{0}
$$

Eq. (2.11) may be represented diagrammatically by Fig. 1, where the terms (a) - (e) represent respectively the five terms on the right-hand side of Eq. (2.11). These correspond respectively to the diagrams (a), (d), (m), (n), and (o) of Fig. 1 of I. We note again only that the diagrams are "time-ordered" in the sense of Goldstone perturbation theory, and that lines with arrows pointing upwards (downwards) are to be strictly associated with particle (hole) states respectively. It is clear by straightforward iteration of Eq. (2.11) in the form expressed diagrammatically in Fig. 1, that the diagrams (c) and (d) taken individually generate respectively the complete sets of ladder diagrams for two-particle scattering and two-hole scattering in the many-body medium. These terms are hence referred to as the ppLAD and hhLAD terms respectively. Similarly the quadratic term of diagram (e) of Fig. 1 generates the mixed particle-particle and hole-hole ladder terms and is hence referred to as the MLAD term.

In order to make further simplifications we introduce relative and centre-ofmass momentum variables,

$$
\begin{gathered}
\vec{P} \equiv \vec{k}_{1}+\vec{k}_{2}=\vec{k}_{1}^{\prime}+\vec{k}_{2}^{\prime}, \\
\vec{p}^{\prime} \equiv \frac{1}{2}\left(\vec{k}_{1}-\vec{k}_{2}\right), \quad \vec{p} \equiv \frac{1}{2}\left(\vec{k}_{1}^{\prime}-\vec{k}_{2}^{\prime}\right)=\vec{p}^{\prime}+\vec{q},
\end{gathered}
$$

where

$$
\vec{k}_{1}^{\prime} \equiv \vec{k}_{1}+\stackrel{\vec{q}}{ }, \quad \vec{k}_{2}^{\prime} \equiv \vec{k}_{2}-\vec{q} .
$$

Using Galilean invariance to write the potential in the form

$$
\left\langle\frac{1}{2} \vec{P}+\vec{p} ; \frac{1}{2} \vec{P}-\vec{p}|V| \frac{1}{2} \vec{P}+\vec{p}^{\prime} ; \frac{1}{2} \vec{P}-\vec{p}^{\prime}\right\rangle \equiv\left\langle\vec{p}|V| \vec{p}^{\prime}\right\rangle
$$


independent of $\vec{P}$, and making the further change of variables

$$
\vec{q}^{\prime} \equiv \vec{k}-\vec{p}^{\prime}, \quad \vec{k} \equiv \vec{k}^{\prime}-\vec{p}^{\prime},
$$

Eq. (2.11) may be rewritten in the form

$$
\begin{aligned}
& \frac{\hbar^{2}}{m}\left(p^{2}-p^{\prime 2}\right) S_{2 ; \vec{p} \vec{p}^{\prime}}^{s_{1} s_{2}}(\vec{P})+\left\langle\vec{p}|V| \vec{p}^{\prime}\right\rangle-\left\langle\vec{p}|V|-\vec{p}^{\prime}\right\rangle \delta_{s_{1} s_{2}} \\
& \quad+\sum_{\vec{k}}\langle\vec{p}|V| \vec{k}\rangle S_{2 ; \vec{k} \vec{p}^{\prime}}^{s_{1} s_{2}}(\vec{P}) \vec{Q}(\vec{k}, \vec{P})+\sum_{\vec{k}^{\prime}} S_{2 ; \vec{p} \vec{k}^{\prime}}^{s_{1} s_{2}},(\vec{P})\left\langle\vec{k}^{\prime}|V| \vec{p}^{\prime}\right\rangle Q\left(\vec{k}^{\prime}, \vec{P}\right) \\
& \quad+\left(1-\frac{1}{2} \delta_{s_{1} s_{2}}\right) \sum_{\vec{k} \vec{k}^{\prime}} S_{2 ; \vec{p} \vec{k}^{\prime}}^{s_{1} s_{2}}(\vec{P})\left\langle\vec{k}^{\prime}|V| \vec{k}\right\rangle S_{2 ; \vec{k} \vec{p}^{\prime}}^{s_{1} s_{2}}(\vec{P}) Q(\vec{k}, \vec{P}) \bar{Q}\left(\vec{k}^{\prime}, \vec{P}\right)=0,
\end{aligned}
$$

where we have introduced the notations

$$
\begin{aligned}
\left\langle\frac{1}{2} \vec{P}+\vec{p}, s_{1} ; \frac{1}{2} \vec{P}-\vec{p}, s_{2}\left|S_{2}\right| \frac{1}{2} \vec{P}+\vec{p}^{\prime}, s_{1} ; \frac{1}{2} \vec{P}-\vec{p}^{\prime}, s_{2}\right\rangle_{A} & \equiv S_{2 ; \vec{p}^{\prime}}^{s_{1} s_{2}}(\vec{P}), \\
\bar{n}\left(\frac{1}{2} \vec{P}+\vec{p}\right) \bar{n}\left(\frac{1}{2} \vec{P}-\vec{p}\right) \equiv \bar{Q}(\vec{p}, \vec{P}), \quad n\left(\frac{1}{2} \vec{P}+\vec{p}^{\prime}\right) n\left(\frac{1}{2} \vec{P}-\vec{p}^{\prime}\right) & \equiv Q\left(\vec{p}^{\prime}, \vec{P}\right) .
\end{aligned}
$$

Finally we can make the antisymmetry of $S_{2}$ explicit by defining the non antisymmetrised quantity

$$
\left\langle\frac{1}{2} \vec{P}+\vec{p}, s_{1} ; \frac{1}{2} \vec{P}-\vec{p}, s_{1}\left|S_{2}\right| \frac{1}{2} \vec{P}+\vec{p}^{\prime}, s_{1} ; \frac{1}{2} \vec{P}-\vec{p}^{\prime}, s_{1}\right\rangle \equiv T_{\vec{p} \vec{p}^{\prime}}(\vec{P}),
$$

which, as written, is independent of spin component $s_{1}$ for a system as considered here where there are no externally applied "magnetic fields" (or, more precisely, where the exact g.s. is assumed to share the invariance under spatial reflections of the Hamiltonian). In this case, by the definitions (2.17) and (2.18), we have

$$
S_{2 ; \tilde{p} \vec{p}^{\prime}}^{S_{1}}(\vec{P})=T_{\vec{p} \vec{p}^{\prime}}(\vec{P})-\delta_{s_{1} s_{2}} T_{\vec{p},-\dot{p}^{\prime}}(\vec{P})
$$

and we have only to consider the two cases of parallel $\left(s_{1}=s_{2}\right)$ and antiparallel $\left(s_{1}=-s_{2}\right)$ spins. It is readily verified from Eq. (2.16) that both the parallel and antiparallel spin cases are satisfied with the same $T$ function in Eq. (2.19), and that the basic CLAD equation for it is now

$$
\begin{aligned}
& \frac{\hbar^{2}}{m}\left(p^{2}-p^{\prime 2}\right) T_{\vec{p} \vec{p}^{\prime}}(\vec{P})+\left\langle\vec{p}|V| \vec{p}^{\prime}\right\rangle \\
& \quad+\sum_{\vec{k}}\langle\vec{p}|V| \vec{k}\rangle T_{\vec{k} \vec{p}^{\prime}}(\vec{P}) \bar{Q}(\vec{k}, \vec{P})+\sum_{\vec{k}^{\prime}} T_{\vec{p} \vec{k}^{\prime}}(\vec{P})\left\langle\vec{k}^{\prime}|V| \vec{p}^{\prime}\right\rangle Q\left(\vec{k}^{\prime}, \vec{P}\right) \\
& \quad+\sum_{\vec{k} \vec{k}^{\prime}} T_{\vec{p} \vec{k}^{\prime}}(\vec{P})\left\langle\vec{k}^{\prime}|V| \vec{k}\right\rangle T_{\vec{k} \vec{p}^{\prime}} \bar{Q}(\vec{k}, \vec{P}) Q\left(\vec{k}^{\prime}, \vec{P}\right)=0 .
\end{aligned}
$$

We note finally that a knowledge of $S_{2}$ is sufficient to determine the g.s. energy $E$. Taking the inner product of Eq. (2.8) with $\langle\Phi|$ readily yields

$$
E=\sum_{v}\left\langle v\left|T \Psi_{1}\right| v\right\rangle+\frac{1}{2} \sum_{v v^{\prime}}\left\langle v v^{\prime}\left|V \Psi_{2}\right| v v^{\prime}\right\rangle_{A} .
$$

In our case it is easy to show that this reduces to the expression

$$
\begin{aligned}
E= & 2 \sum_{\vec{k}} \frac{\hbar^{2} k^{2}}{2 m} n(k)+\sum_{\vec{p}^{\prime} \vec{P}} Q\left(\vec{p}^{\prime}, \vec{P}\right)\left[2\left\langle\vec{p}^{\prime}|V| \vec{p}^{\prime}\right\rangle-\left\langle\vec{p}^{\prime}|V|-\vec{p}^{\prime}\right\rangle\right. \\
& \left.+\sum_{\vec{p}}\left\langle\vec{p}^{\prime}|V| \vec{p}\right\rangle\left\{2 T_{\vec{p}^{\prime}}(\vec{P})-T_{\vec{p},-\vec{p}^{\prime}}(\vec{P})\right\} \bar{Q}(\vec{p}, \vec{P})\right],
\end{aligned}
$$

where the factors of 2 are from the spin sums. 
In the thermodynamic limit in which we are interested where $N \rightarrow \infty, \Omega \rightarrow \infty$, and $\rho=N / \Omega$ remains finite, the single particle sums are replaced by integrals in the usual way,

$$
\sum_{\vec{k}} \rightarrow \Omega(2 \pi)^{-3} \int d \vec{k},
$$

and hence as usual for a spin-saturated spin- $\frac{1}{2}$ system the Fermi momentum $k_{F}$ is given by $\rho=k_{F}^{3} / 3 \pi^{2}$.

In the succeeding paper [29] we shall now consider solving the CLAD equation (2.21) for the special case of separable potentials.

\section{Final Remarks}

We have seen how at the so-called SUB2 level of truncation, which is the lowest natural level of approximation for homogeneous systems, the CCF may be reduced to a nonlinear (-in fact, bilinear) integral equation for the four-point correlation function, $S_{2}$. This correlation function is interpreted as the wavefunction amplitude which fully incorporates the pairing substructure within the many-body system. More specifically, it provides a measure of the linked two-particle/two-hole component in the true "ground-state" wavefunction. The SUB2 approximation includes in a self-consistent fashion the effects of particle-particle, hole-hole, and particle-hole correlations, all of which are included on an essentially equal footing. By contrast to an earlier work I where we focussed attention on particle-hole correlations and the associated random-phase approximation (RPA), we concentrate here on the particle-particle/hole-hole channels and the associated complete ladder (CLAD) approximation.

We note that both the RPA equation, given by Eqs. (3.10)-(3.12) of I, and the present CLAD equation (2.16) for $S_{2}$, are bilinear integral equations. By suitably extending the techniques developed in I for exactly solving the RPA equations for local potentials, we shall show in the succeeding paper [29] how the CLAD equation (2.16) may also be exactly solved for non-local potentials of the separable kind.

It has sometimes come as a surprise to readers not familiar with the CCF that such well-known and simple theories as the RPA should be so much more cumbersome to re-derive within the CCF, than within such familiar alternative techniques as those employing Green's functions [5], for example. In particular, one needs to address the question as to why the RPA, for example, should be represented by a nonlinear equation for $S_{2}$, when the corresponding underlying physical approximation is generally regarded as being intrinsically linear. The crux of this seeming dichotomy is that the approximation is only linear when formulated in terms of a suitable two-point physical response function in the particle-hole channel of interest. In the Green's function language, this would be the proper polarisation operator, for which the RPA solution is indeed very simple. It is important to realise that, by contrast, we work directly within the CCF with the four-point function, $S_{2}$, which, in principle, contains much more information about the system. Clearly, if one is only interested in the g.s. energy of the system, this additional information is not needed. In diagrammatic terms it is straightforward to show that no linear equation for $S_{2}$ could ever incorporate the effects of the important "backward- 
going" bubble diagrams included in the RPA beyond the purely "forward-going" analogous terms included in the Tamm-Dancoff approximation (TDA). It is necessary to include $S_{2}$ at least bilinearly to achieve this, and we see that the SUB2 approximation is actually "minimally nonlinear" in this regard.

In the present paper we have stressed the links between the CCF and (timeindependent) perturbation theory. It is however perhaps worth pointing out that the CCF may also be cast in a variational form, although we stress from the outset that in the form employed here the method is certainly not fully variational in the usual Rayleigh-Ritz sense of providing upper bounds for the energy, for example. An obvious reason for this is that we have calculated the g.s. energy, $E$, as in Eq. (2.22), from the expression $E=\langle\Phi|H| \Psi\rangle=\left\langle\Phi\left|H e^{S}\right| \Phi\right\rangle=\left\langle\Phi\left|e^{-S} H e^{S}\right| \Phi\right\rangle$, rather than from the direct expectation value, $E=\langle\Psi|H| \Psi\rangle\langle\langle\Psi \mid \Psi\rangle$, which is necessary for the energy variational principle. It is clear that although expectation values of arbitrary operators, rather than the Hamiltonian, would need to be calculated using both the ket state $|\Psi\rangle=e^{S}|\Phi\rangle$ and the bra state $\langle\Psi|=\langle\Phi| e^{S^{\dagger}}$, for the g.s. energy we have been able to employ the Schrödinger equation to evaluate $E$ using the ket state only.

The above consideration is actually more important than it might seem. Thus, the CCF is very fundamentally built upon the similarity transformation $H \rightarrow e^{-S} H e^{S}$, as in Eq. (2.9) and its subsequent discussion. By contrast, direct expectation values require the analogous operator $e^{S t} H e^{S}$ which is much more complicated and less amenable to evaluation. In particular, this latter expression has no simple, finite expansion comparable to the well-known nested commutator expansion for the expression $e^{-S} H e^{S}$. Given that $S$ is built out of creation operators only with respect to the state $|\Phi\rangle$ (i.e., operators that create holes within or particle states outside the filled Fermi sea), this otherwise infinite nested commutator expansion truncates exactly at the term involving the $2 m$-fold nested commutator, $[\cdots[H, S], \cdots, S]$, if $H$ is a sum of $m$-body operators. It is this underlying similarity transformation that leads to finite equations within the CCF. By contrast, arbitrary expectation values have infinite expansions in terms of $S$ and $S^{\dagger}$, even within a given SUB $n$ truncation.

If one is interested in operators other than $H$, the above consideration is apparently a disadvantage of the CCF as described here. We point out that this disadvantage may be rather effectively removed by using a recent extended version of the CCF, as developed by Arponen [43] and as discussed and applied at length by one of the present authors and his collaborators [44-46]. In this extended version the ket state is still represented by $|\Psi\rangle=e^{S}|\Phi\rangle=e^{S} e^{-S^{\prime \prime}}|\Phi\rangle$, but it is now shown how the bra state may be represented as $\langle\Psi|=\langle\Phi| e^{S^{\prime \prime}} e^{-S}$, with both operators $S$ and $S^{\prime \prime}$ composed of fully-linked pieces only. Furthermore, just as $S$ contains only creation operators with respect to $|\Phi\rangle$, as in Eqs. (2.5)-(2.6), so $S^{\prime \prime}$ now contains only destruction operators with respect to $|\Phi\rangle$. This extended version of the CCF is then built fundamentally on a comparable double similarity transformation, $A \rightarrow e^{S^{\prime \prime}} e^{-S} A e^{S} e^{-S^{\prime \prime}}$, for an arbitrary operator $A$.

A discussion of this extended version of the CCF would take us too far afield for present purposes. The interested reader is referred to refs. [43-45] for further details. We comment only that the resulting formulation of the quantum many-body problem is essentially a biorthogonal one, and it is shown $[44,47]$ how it may be derived in its most general time-dependent form from a variational principle for the 
action. Even in the extended version most practical truncation schemes still do not however lead to variational bounds for the energy, since the truncations on the operators $\left\{S, S^{\prime \prime}\right\}$ do not generally lead to the states $\langle\Psi|$ and $|\Psi\rangle$ being Hermitian conjugates of each other as required by the energy variational principle.

A comparable variational formulation of the normal CCF (i.e., in the form as employed here) is also possible. Indeed, one of the present authors and his collaborators have given [47] a unified treatment (and a comparison) of the socalled configuration-interaction method [48], the (normal) CCF employed here, and the extended CCF. In particular, it is shown how each formulation may be derived by a specific parametrisation of the g.s. bra and ket wavefunctions in the action functional. Contact is made in each case with time-independent perturbation theory, and the various "tree-diagram" structures that emerge in each theory are discussed. The relationships of the CCF, both in its normal form employed here and in its more recent extended form, with Green's function and other generalised equation-of-motion techniques, are also touched upon in refs. [44, 45, 47].

Finally, we point out that the excited states within the CCF will be discussed, both generally and very specifically for the separable model potential considered in the subsequent paper [29], in a third paper [30] in this series. A comparable general discussion of excited states within the extended version of the CCF has also been given recently [45].

Acknowledgements. This work was largely supported by the Science and Engineering Research Council of Great Britain, and in part by the Polish-U.S. Maria Sklodowska-Curie fund. Both of their supports are gratefully acknowledged. One of us (W.P.) would like to thank the Department of Mathematics at UMIST for their warm and generous hospitality during his stay there. W.P. also acknowledges stimulating discussions with Professor J. Dabrowski.

\section{References}

1. Brueckner, K. A., Levinson, C. A., Mahmoud, H. M.: Phys. Rev. 95, 217 (1954)

2. Landau, L. D.: Zh. Eksp. Teor. Fiz. 30, 1058 (1956) [Sov. Phys. JETP 3, 920 (1956)]; 32, 59 (1957) $[5,101(1957)]$

3. Goldstone, J.: Proc. R. Soc. Lond. A239, 267 (1957)

4. Cooper, L. N.: Phys. Rev. 104, 1189 (1956)

5. Galitskii, V. M., Migdal, A. B.: Zh. Eksp. Teor. Fiz. 34, 139 (1958) [Sov. Phys. JETP 7, 96 (1958)]; Galitskii, V. M.: Zh. Eksp. Teor. Fiz. 34, 151 (1958) [Sov. Phys. JETP 7, 104 (1958)]

6. Bishop, R. F.: Ann. Phys. (NY) 77, 106 (1973)

7. Brueckner, K. A., Levinson, C. A.: Phys. Rev. 97, 1344 (1955); Brueckner, K. A.: Phys. Rev. 100, $36(1955)$

8. Bethe, H. A., Goldstone, J.: Proc. R. Soc. Lond. A238, 551 (1957)

9. Salpeter, E. E., Bethe, H. A.: Phys. Rev. 84, 1232 (1951)

10. Chisholm, J. S. R., Squires, E. J.: Nucl. Phys. 13, 156 (1959)

11. Mehta, M. L.: Nucl. Phys. 12, 333 (1959)

12. Mehta, M. L.: Nucl. Phys. 20, 533 (1960)

13. Bishop, R. F., Strayer, M. R., Irvine, J. M.: Phys. Rev. A10, 2423 (1974)

14. Bishop, R. F., Strayer, M. R., Irvine, J. M.: J. Low Temp. Phys. 20, 573 (1975)

15. Lippmann, B. A., Schwinger, J.: Phys. Rev. 79, 469 (1950)

16. Ghassib, H. B., Bishop, R. F., Strayer, M. R.: J. Low Temp. Phys. 23, 393 (1976) 
17. Bishop, R. F., Ghassib, H. B., Strayer, M. R.: Phys. Rev. A13, 1570 (1976)

18. Bishop, R. F.: Ann. Phys. (NY) 99, 318 (1976)

19. Bishop, R. F., Strayer, M. R.: J. Phys. G 3, L13 (1977)

20. Breit, G., Bouricius, W. G.: Phys. Rev. 75, 1029 (1949)

21. Feshbach, H., Lomon, E.: Phys. Rev. 102, 891 (1956)

22. Kohn, W., Sherrington, D.: Rev. Mod. Phys. 42, 1 (1970)

23. Yang, C. N.: Rev. Mod. Phys. 34, 694 (1962)

24. Coester, F.: Nucl. Phys. 7, 421 (1958)

25. Coester, F., Kümmel, H.: Nucl. Phys. 17, 477 (1960)

26. Coester, F.: in: Lectures in Theoretical Physics, Vol. 11 (Mahanthappa, K. H., ed.). New York: Gordon and Breach 1969

27. Kümmel, H.: Nucl. Phys. A176, 205 (1971)

28. Kümmel, H., Lührmann, K. H.: Nucl. Phys. A191, 525 (1972)

29. Bishop, R. F., Piechocki, W., Stevens, G. A.: Few-Body Systems 4, 179 (1988)

30. Bishop, R. F., Piechocki, W., Stevens, G. A.: to be published

31. Bishop, R. F., Lührmann, K. H.: Phys. Rev. B17, 3757 (1978)

32. Bishop, R. F., Lührmann, K. H.: Phys. Rev. B26, 5523 (1982)

33. Bishop, R. F., Lührmann, K. H.: Physica 108B, 873 (1981)

34. Bishop, R. F., Lührmann, K. H.: Physica 108B, 1383 (1981)

35. Bishop, R. F.: in: Recent Progress in Many-Body Theories. Proc. of the 2nd Int. Conf., Oaxtepec, Mexico, 1981 (Lecture Notes in Physics, Vol. 142), (Zabolitzky, J. G., et al., eds.), p. 111. BerlinHeidelberg-New York: Springer 1981

36. Macke, W.: Z. Naturforsch. A5, 192 (1950)

37. Tamm, I.: J. Phys. (USSR) 9, 449 (1945); Dancoff, S. M.: Phys. Rev. 78, 382 (1950)

38. Bohm, D., Pines, D.: Phys. Rev. 92, 609 (1953)

39. Gell-Mann, M., Brueckner, K. A.: Phys. Rev. 106, 364 (1957)

40. Bethe, H. A., Brandow, B. H., Petschek, A. G.: Phys. Rev. 129, 225 (1963); Brandow, B. H.: Phys. Rev. 152, 863 (1966)

41. Dabrowski, J.: Can. J. Phys. 61, 1448 (1983)

42. Lührmann, K. H., Kümmel, H.: Nucl. Phys. A194, 225 (1972)

43. Arponen, J.: Ann. Phys. (NY) 151, 311 (1983)

44. Arponen, J. S., Bishop, R. F., Pajanne, E.: Phys. Rev. A36, 2519 (1987)

45. Arponen, J. S., Bishop, R. F., Pajanne, E.: Phys. Rev. A36, 2539 (1987)

46. Arponen, J., Bishop, R. F., Pajanne, E., Robinson, N. I.: Phys. Rev. A37, 1065 (1988)

47. Arponen, J., Bishop, R. F., Pajanne, E.: in: Condensed Matter Theories, Vol. 2 (Vashishta, P., Kalia, R. K., Bishop, R. F., eds.), p. 357. New York: Plenum 1987

48. Nesbet, R. K.: Phys. Rev. 109, 1632 (1958)

Received March 7, 1988; accepted for publication April 12, 1988 